\title{
Vorhofflimmern - Diagnostik
}

\author{
Jens Jung, Michael Böhm
}

Medizinische Klinik und Poliklinik, Innere Medizin III (Kardiologie/Angiologie), Universitätskliniken des Saarlandes, Homburg/Saar

Vorhofflimmern stellt mit einer Prävalenz von 0,4-0,9\% der Erwachsenenbevölkerung die häufigste anhaltende Herzrhythmusstörung dar. Es wird geschätzt, dass in der Bundesrepublik Deutschland etwa 600000 Menschen betroffen sind. Die Häufigkeit dieser Arrhythmie nimmt in Abhängigkeit vom Alter ab dem 40 . Lebensjahr um etwa $0,1-0,2 \%$ pro Jahr $z u$ und liegt bei der Bevölkerungsgruppe über 60 Jahre bei 2-4\% $(16,21)$. Neben dem Alter begünstigen insbesondere kardiovaskuläre Begleiterkrankungen das Auftreten von Vorhofflimmern. Bei etwa 25\% der Patienten mit Vorhofflimmern besteht eine koronare Herzerkrankung (16). Patienten mit akutem Myokardinfarkt haben in 10-11\% Vorhofflimmern $(12,22)$. Eine rheumatische Klappenerkrankung erhöht das Risiko, Vorhofflimmern zu entwickeln, um das 1,8-Fache (5). Die Prävalenz von Vorhofflimmern kann bei Patienten mit einer manifesten Herzinsuffizienz bis zu $40 \%$ erreichen (5).

Vorhofflimmern kann den frühen postoperativen Verlauf nach kardiochirurgischen Eingriffen bei etwa 30\% der Patienten komplizieren (4). Auch andere bradykarde und tachykarde Herzrhythmusstörungen können mit Vorhofflimmern assoziiert sein. An bradykarden Rhythmusstörungen wäre insbesondere das Sinusknotensyndrom zu nennen, bei dem in bis zu $28 \%$ mit atrialen Tachyarrhythmien zur rechnen ist (1). Bei Patienten mit WPW-Syndrom besteht in bis zu 30\% ein begleitendes Vorhofflimmern (10). Auch bei Patienten mit AV-Knoten-
Reentry-Tachykardien oder atrialen Tachykardien kann nicht selten ein begleitendes Vorhofflimmern dokumentiert werden.

Neben einer kardialen Erkrankung können auch extrakardiale Begleiterkrankungen mit einem erhöhtem Risiko für Vorhofflimmern vergesellschaftet sein. So besteht bei Patienten mit einer arteriellen Hypertonie ein 1,5-fach erhöhtes und bei Patienten mit Diabetes mellitus ein 1,4-fach erhöhtes Risiko Vorhofflimmern zu entwickeln (6). Patienten mit chronisch obstruktiver Lungenerkrankung leiden in etwa 3\% an begleitendem Vorhofflimmern. Eine hyperthyreote Stoffwechsellage als Ursache von Vorhofflimmern liegt in etwa $2 \%$ vor (18).

Bei etwa 3-11\% der Patienten mit Vorhofflimmern besteht jedoch keine kardiale Grunderkrankung bzw. prädisponierende extrakardiale Begleiterkrankung, so dass von einem sogenannten "idiopathischen" Vorhofflimmern auszugehen ist $(8,17)$. Bei einem Teil dieser Patienten, vorwiegend Männer in der vierten Lebensdekade, treten die Arrhythmieepisoden ausschließlich in der Ruhephase und häufig in der Nacht auf, so dass ein vagal getriggertes Vorhofflimmern vorliegt (11).

kurzgefasst: Vorhofflimmern stellt die häufigste anhaltende Arrhythmie dar mit steigender Prävalenz in höherem Alter bzw. bei kardiovaskulären Grunderkrankungen.

\section{Vorhofflimmern - Der konkrete Fall}

Anamnnese: Ein 56-jähriger Patient suchte aufgrund einer seit einigen Tagen bestehenden Einschränkung der körperlichen Belastbarkeit verbunden mit Palpitationen in Form eines inneren Unruhegefühls seinen Hausarzt auf. Dieser stellte die Diagnose eines neu aufgetretenen Vorhofflimmerns und wies den Patienten in die Klinik ein. Der genaue Zeitpunkt des Beginns der Arrhythmieepisode ließ sich nicht eruieren. Es handelte sich um ein erstmaliges Ereignis. Außerhalb der aktuellen Beschwerden bestand bisher eine normale Leistungsbreite ohne Belastungsdyspnoe oder pektanginöse Beschwerden. Bis auf eine mit einem ACE-Hemmer therapierte arterielle Hypertonie bestanden keine weiteren Vorerkrankungen.

Untersuchumgen: Bei der körperlichen Untersuchung zeigten sich keine kardialen Dekompensationszeichen. Auskultato- risch fanden sich unregelmäßige Herzaktionen, kein vitientypischer Auskultationsbefund. Elektrokardiographisch konnte eine Tachyarrhythmia absoluta bei Vorhofflimmern mit einer Ventrikelfrequenz von 110-140 Schlägen/Minute dokumentiert werden. Es bestand eine linkstypische Herzachse bei unauffälligem Stromkurvenverlauf. Röntgenologisch war das Herz normal dimensioniert. Kein Nachweis von Stauungszeichen oder pneumonischen Infiltraten. Laborchemisch konnten eine Hyperthyreose sowie eine Elektrolytentgleisung ausgeschlossen werden. Echokardiographisch ergab sich nach Frequenzlimitierung bis auf eine geringe Dilatation des linken Vorhofes auf $43 \mathrm{~mm}$ kein auffälliger Befund. Bei der nach Rhythmisierung erfolgten fahrradergometrischen Belastung zeigten sich keine Hinweise auf eine koronare Herzerkrankung.
Therapie und Verlauf: Zur Thromboembolieprophylaxe wurde eine therapeutische Heparinisierung eingeleitet. Nach intravenöser Gabe von Metoprolol konnte eine Normalisierung der Herzfrequenz erreicht werden. Nach Ausschluss atrialer Thromben in der transösophagealen Echokardiographie wurde unter Beibehaltung der Antikoagulation eine elektrische Kardioversion in tiefer Sedierung durchgeführt. Nach Applikation von 200 J konnte der Sinusrhythmus wiederhergestellt werden. Unter überlappender Heparinisierung wurde der Patient auf Phenprocoumon eingestellt (INR 2-3). Die orale Antikoagulation wurde bei stabilem Sinusrhythmus über 4 Wochen fortgeführt. Wegen des erstmaligen Auftretens des Vorhofflimmerns wurde zunächst von einer rezidivprophylaktischen antiarrhythmischen Therapie abgesehen. J. Jung, M. Böhm, Homburg/Saar 


\section{Pathophysiologie}

Pathologisch-anatomische Veränderungen des Vorhofmyokards wie Fibrose oder inflammatorische Prozesse stellen das Substrat für die Entwicklung von Vorhofflimmern dar (13). Als potenzielle Triggerfaktoren für die Initiierung der $\mathrm{Ar}$ rhythmie können neben Veränderungen des autonomen Tonus (11) bzw. einer akuten oder chronischen Vorhofbelastung (23) auch ektope Foci dienen (15).

Als wesentliches pathophysiologisches Prinzip zur Aufrechterhaltung von Vorhofflimmern werden multiple kreisende Erregungen im Vorhofmyokard angesehen (19). Die ersten theoretischen Überlegungen wurden anhand von MappingUntersuchungen am Tiermodell bestätigt (3). Im Rahmen dieser Untersuchungen konnte gezeigt werden, dass etwa 6 Wiedereintrittskreise notwendig sind, um Vorhofflimmern aufrechterhalten zu können.

Vorhofflimmern selbst führt zu Veränderungen am Vorhofmyokard, die zu einer Aufrechterhaltung der Arrhythmie beitragen, die mit dem Begriff »electrical remodeling" zusammengefasst werden. Hier spielen eine Verkürzung der atrialen Refraktärperioden sowie eine intrazelluläre Calciumüberladung atrialer Myozyten eine wesentliche Rolle (2).

In jüngster Zeit wurde familiär gehäuft auftretendes Vorhofflimmern assoziiert mit einem Gendefekt auf Chromosom 10 beschrieben (9).

kurzgefasst: Multiple kreisende Erregungsfronten in den Vorhöfen sind die pathophysiologische Grundlage von Vorhofflimmern. Die Arrhythmie selbst führt zu Umbauprozessen, die zu ihrer Perpetuierung beitragen.

\section{Klassifikation}

Vorhofflimmern lässt sich aufgrund seiner klinischen Erscheinungsform klassifizieren (14). Das erstmalige Auftreten der Arrhythmie wird als akutes Vorhofflimmern bezeichnet. Bei der chronischen Verlaufsform werden drei Manifestationsmöglichkeiten unterschieden. Als paroxysmales Vorhofflimmern wird rezidivierendes anfallsartiges Vorhofflimmern bezeichnet, das spontan innerhalb von 24 Stunden terminiert. Von persistierendem Vorhofflimmern spricht man bei länger als 24 Stunden anhaltenden Arrhythmieepisoden, die nicht spontan in Sinusrhythmus konvertieren und daher durch eine Kardioversion zu terminieren sind. Wenn Vorhofflimmern nicht mehr in Sinusrhythmus zu konvertieren ist, liegt permanentes Vorhofflimmern vor. Diese Verlaufsform wird im klinischen Alltag häufig auch als chronisches Vorhofflimmern bezeichnet.

\section{Klinischer Verlauf und Prognose}

Bei Patienten mit anfallsartigem Vorhofflimmern kommt es in etwa $12 \%$ im weiteren Verlauf zu einem permanenten Vorhofflimmern. In älteren Untersuchungen konnte gezeigt werden, dass die Morbidität und Mortalität bei Patienten mit Vorhofflimmern erhöht sind. So war in der Framingham-Studie Vorhofflimmern mit einer im Vergleich zur Kontrollgruppe verdoppelten Mortalitätsrate vergesellschaftet (7). Neben einer erhöhten Rate an thromboembolischen Ereignissen, insbesondere an Schlaganfällen, tragen die mit Vorhofflimmern as- soziierten kardiovaskulären Begleiterkrankungen und das oft höhere Lebensalter der Patienten zu einer erhöhten Mortalität bei. Durch den Einsatz einer verbesserten Therapie bei Patienten mit Herzinsuffizienz und Vorhofflimmern konnte die Prognose dieser Patienten in den letzten Jahren erheblich gebessert werden (24). Ob Vorhofflimmern per se unabhängig von dem Alter der Patienten und der kardiovaskulären Begleiterkrankungen mit einer erhöhten Mortalität behaftet ist, ist nicht sicher belegt.

kurzgefasst: Die Prognose von Patienten mit Vorhofflimmern wird im Wesentlichen durch thromboembolische Komplikationen und die kardiovaskulären Begleiterkrankungen bestimmt.

\section{Symptomatik}

Die Symptomatik von Vorhofflimmern kann erheblich variieren. Neben asymptomatischen bis oligosymptomatischen Verläufen gibt es andere, die insbesondere zu Beginn einer Arrhythmieepisode hochsymptomatisch sind. Im Vordergrund der Beschwerden stehen Palpitationen, eingeschränkte körperliche Leistungsbreite, Schwindel und seltener auch Synkopen. Jedoch nicht alle Episoden von Vorhofflimmern eines Patienten gehen mit klinischen Beschwerden einher. In Langzeit-EKG-Untersuchungen hatten Patienten mit paroxysmalem Vorhofflimmern häufiger asymptomatische Episoden als symptomatische (20). Die klinische Symptomatik wird wesentlich durch die hämodynamischen Effekte beeinflusst. Der Verlust des Vorhofbeitrages zur ventrikulären Füllung ist mit einer etwa 20\%-igen Abnahme des Schlagvolumens vergesellschaftet. Die irreguläre Kammererregung trägt ebenfalls zu einer Verminderung der kardialen Förderleistung bei. Bei Patienten mit persistierender Tachyarrhythmie kann sich eine tachysystolische Kardiomyopathie ausbilden mit progredienten Zeichen der Herzinsuffizienz.

kurzgefasst: Das typische Beschwerdebild bei Patienten mit Vorhofflimmern umfasst Palpitationen, eingeschränkte körperliche Leistungsbreite, Schwindel und seltener auch Synkopen.

\section{Klinische Untersuchungsmethoden}

Die Abklärung von Vorhofflimmern bei Patienten bietet im Wesentlichen zwei Aspekte. Zum Einen handelt es sich häufig um eine symptomatische Arrhythmie, so dass die Abklärung des Beschwerdebildes und damit die Diagnosestellung im Vordergrund steht. Zum Anderen muss bei dokumentiertem Vorhofflimmern nach einer hiermit assoziierten Begleiterkrankung gefahndet werden.

Die Anamnese zielt darauf ab, das klinische Beschwerdebild einzugrenzen. Fragen nach der klinischen Symptomatik, den Umständen des Auftretens von Vorhofflimmerepisoden, Dauer und Häufigkeit sowie der Modus der Terminierung der Arrhythmie z.B. spontan oder nach medikamentöser bzw. elektrischer Kardioversion stehen hierbei im Vordergrund. Weiterhin sollte nach Symptomen einer zugrundeliegenden kardialen Erkrankung wie beispielsweise Angina pectoris oder Belastungsdyspnoe und nach mit Vorhofflimmern assoziierten Risikofaktoren wie Diabetes mellitus oder arterieller Hypertonie gefragt werden. Die körperliche Untersuchung dient zur Erfassung einer kardialen oder extrakardialen Begleiterkrankung. 
Im Ruhe-EKG ist Vorhofflimmern durch unregelmäßige Abstände der QRS-Komplexe gekennzeichnet. Die Grundlinie weist feine bis grobe unregelmäßige Flimmerwellen mit einer Frequenz von 350-600/min. auf. Die Flimmerwellen sind meist in der Ableitung V1 am besten zu erkennen. Vorhofflimmern mit regelmäßigen Abständen der QRS-Komplexe bei langsamer Ventrikelfrequenz tritt bei Patienten mit totalem AV-Block und junktionalem oder ventrikulärem Ersatzrhythmus auf. Neben der Diagnosestellung bietet das Ruhe-EKG die Möglichkeit, elektrokardiographische Hinweise auf eine zugrundeliegende kardiale Erkrankung (Hypertrophiezeichen, Infarktnarbe etc.) zu erfassen.

Mittels Langzeit-EKG können spontan auftretende paroxysmale Episoden von Vorhofflimmern registriert werden. Hier steht die Abklärung von Symptomen, die an ein intermittierendes Vorhofflimmern denken lassen, im Vordergrund. Zudem können langzeitelektrokardiographisch eventuell vorhandene Triggermechanismen wie atriale Extrasystolen oder durch Bradykardie induziertes Vorhofflimmern erfasst werden.

Ein Belastungs-EKG sollte bei der Abklärung von intermittierendem Vorhofflimmern zum nicht-invasiven Ausschluss einer koronaren Herzerkrankung durchgeführt werden. Bei Patienten mit belastungsabhängigen Palpitationen dient die Ergometrie zur Erfassung von sympathikoton getriggertem Vorhofflimmern. Zum Ausschluss einer kardialen Grunderkrankung ist ergänzend eine Echokardiographie indiziert. Laborchemische Untersuchungen sollten eine Bestimmung des basalen TSHWertes und der Elektrolyte umfassen.

kurzgefasst: Die Basisdiagnostik bei Patienten mit Vorhofflimmern umfasst neben der Anamnese und der körperlichen Untersuchung eine kardiale Funktionsdiagnostik einschließlich Echokardiographie und Belastungs-EKG sowie die Bestimmung von basalem TSH und den Elektrolyten.

\section{Literatur}

1. Alboni P, Menozzi C, Brignole M, et al. Effects of permanent pacemaker and oral theophylline in sick sinus syndrome. The Theopace Study. Circulation 1997:96:260-266.

2. Allessie MA, Boyden PA, Camm AJ, et al. Pathophysiology and prevention of atrial fibrillation. Circulation 2001;103:769-777.

3. Allessie MA, Lammers WJEP, Bonke FIM, Hollen J. Experimental evaluation of Moe's wavelet hypothesis of atrial fibrillation. In: Zipes DP, Jalife J, eds. Cardiac electrophysiology and arrhythmias. Orlando, Fla: Grune \& Stratton, 1985:265-275.

4. Almassi GH, Schowalter T, Nicolosi AC, Aggarwal A, Moritz TE, Henderson WG, Tarazi R, Shroyer AL, Sethi GK, Grover FL, Hammermeister KE. Atrial fibrillation after cardiac surgery: a major morbid event? Ann Surg 1997;226:501-511.

5. Alpert JS, Petersen P, Godtfredsen J. Atrial fibrillation: natural history, complications and management. Ann Rev Med 1988;39:4152.

6. Benjamin EJ, Levy D, Vaziri SM, Dagostina RB, Belanger AJ, Wolf PA. Independent risk factors for atrial fibrillation in a populationbased cohort: the Framingham Heart Study. JAMA 1994; 271:840-844.

7. Benjamin EJ, Wolf PA, D'Agostino RB, Silbershatz H, Kannel WB, Levy D. Impact of atrial fibrillation on the risk of death: The Framingham study. Circulation 1998;98:946-952.
8. Brand FN, Abbott RD, Kannel WB, Wolf PA. Characteristics and prognosis of lone atrial fibrillation. 30-year follow-up in the Framingham study. JAMA 1985;254:3449-3453.

9. Brugada R, Tapscott T, Czernuszewicz GZ, Marian AJ, Iglesias A, Mont L, Brugada J, Girona J, Domingo A, Bachinski LL, Roberts R. Identification of a genetic locus for familial atrial fibrillation. $\mathrm{N}$ Engl J Med 1997; 336:905-911.

10. Campbell RW, Smith RA, Gallagher JJ, Pritchett EL, Wallace AG. Atrial fibrillation in the preexcitation syndrome. Am J Cardiol 1977;40:514-520.

11. Coumel P. Autonomic influences in atrial tachyarrhythmias. J Cardiovasc Electrophysiol 1996;7:999-1007.

12. Eldar M, Canetti M, Rotstein Z, Boyko V, Gottlieb S, Kaplinsky E, Behar S. Significance of paroxysmal atrial fibrillation complicating acute myocardial infarction in the thrombolytic era. SPRINT and thrombolytic survey groups. Circulation 1998;97:965-970.

13. Falk RH. Etiology and complications of atrial fibrillation: insights from pathology studies. Am J Cardiol 1998;82:10N-17N.

14. Gallagher M, Camm AJ. Classification of atrial fibrillation. PACE 1997;20:1603-1605.

15. Haissaguerre M, Jais P, Shah DC, Takahashi A, Hocini M, Quiniou G, Garrigue S, Le Mouroux A, Le Metayer P, Clementy J. Spontaneous initiation of atrial fibrillation by ectopic beats originating in the pulmonary veins. N Engl J Med 1998;339:659-666.

16. Kannel WB, Abbott RD, Savage DD, McNamara PM. Epidemiologic features of chronic atrial fibrillation: the Framingham study. $\mathrm{N}$ Engl J Med 1982;306:1018-1022.

17. Kopecky SL, Gersh BJ, McGoon MD, Whisnant JP, Holmes DR Jr, Ilstrup DM, Frye RL. The natural history of lone atrial fibrillation: a population based study over three decades. $N$ Engl J Med 1987;317:669-674.

18. Krahn AD, Klein GJ, Kerr CR, Boone J, Sheldon R, Green M, Talajic $\mathrm{M}$, Wang $\mathrm{X}$, Connolly S. How useful is thyroid function testing in patients with recent-onset atrial fibrillation? The Canadian Registry of Atrial Fibrillation Investigators. Arch Intern Med 1996;156:2221-2224.

19. Moe GK. On the multiple wavelet hypothesis of atrial fibrillation. Arch Int Pharmacodyn Ther 1962;140:183-188.

20. Page RL, Wilkinson WE, Clair WK. Asymptomatic arrhythmias in patients with symptomatic atrial fibrillation and paroxysmal supraventricular tachycardia. Circulation 1994;89:224-227.

21. Petersen P, Godtfredsen J. Atrial fibrillation: a review of course and prognosis. Acta Med Scand 1984;216:5-9.

22. Sakata K, Kurihara H, Iwamori K, Maki A, Yoshino H, Yanagisawa A, Ishikawa K. Clinical and prognostic significance of atrial fibrillation in acute myocardial infarction. Am J Cardiol 1997;80:15221527.

23. Satoh T, Zipes DP. Unequal atrial stretch in dogs increases dispersion of refractoriness conductive to developing atrial fibrillation. J Cardiovasc Electrophysiol 1996;7:833-842.

24. Stevenson WG, Stevenson LW, Middlekauff HR, Fonarow GC, Hamilton MA, Woo MA, Saxon LA, Natterson PD, Steimle A, Walden $\mathrm{JA}$, Tillisch JH. Improving survival for patients with atrial fibrillation and advanced heart failure. J Am Coll Cardiol 1996; 28:14581463.

\section{Korrespondenz}

Priv.-Doz. Dr. med. Jens Jung

Medizinische Universitätsklinik und Poliklinik

Innere Medizin III (Kardiologie/Angiologie)

Universitätskliniken des Saarlandes

66421 Homburg/Saar

Tel.: 06841-1623359

Fax: 06841-1623394

E-Mail: jung@med-in.uni-saarland.de 